\title{
EXPERIENCE RATING OF ARIMA PROCESSES BY THE KALMAN FILTER
}

\author{
By JUKKa RANTALA
}

The Ministry of Social Affairs and Health, Helsinki

\begin{abstract}
This paper deals with experience rating of claims processes of ARIMA structures. By experience rating we mean that future premiums should be only a function of past values of the claims process. The main emphasis is on demonstrating the usefulness of the control-theoretical approach in the search for optimal rating rules. Optimality is here defined to mean as smooth a flow of premiums as possible when the variation in the accumulated profit is restricted to a certain amount. First it is shown how the underlying model in its simplest form can be transformed into the state-space form. Then the Kalman filter technique is used to find the optimal rules. Also a time delay in information is taken into account. The optimal rules are illustrated by examples.
\end{abstract}

\section{INTRODUCTION}

Assume that the $d$ th difference $\Delta^{d} X(t)(d \geqslant 0)$ of the annual total claims process $X(t)$ of an insurance portfolio is a weakly stationary process. Let $P(t)$ be the risk premiums to be controlled. Denote by $U(t)$ the solvency margin or equivalently the accumulated profit at the beginning of the year $t$. To simplify notations $\Delta^{d} X(t), \Delta^{d} P(t)$ and $U(t)$ are taken to mean the deviations from the corresponding expectations.

Assume that it is desired to find a linear rating policy $P(s), P(s+1), \ldots, P(N)$ for a finite time span, which will minimize the quadratic performance criterion

$$
E\left\{\sum_{t=s}^{N}\left[U(t)^{2}+v \cdot\left(\Delta^{d} P(t)\right)^{2}\right]\right\}
$$

where $v \geqslant 0$. This criterion may be interpreted to mean the minimization of the variance of $\Delta^{d} P$ subject to a constraint on the variance of $U$, or equivalently minimization of $\operatorname{Var}(U)$ subject to a constraint on $\operatorname{Var}\left(\Delta^{d} P\right)$.

Observe that criterion (1) differs from those traditionally used in experience rating or credibility theory. In credibility theory, interest is usually in maximum accuracy; i.e., premiums $P(t)$ should be a best estimate of $X(t)$; usually in the LSE sense. No weight is placed on the time stability of premiums or insurer's solvency margin. In experience rating theory some attention has been paid to the limited fluctuation criterion, where variance in premiums has to be restricted. However, the starting-point is generally a fixed formula whose optimality properties are usually not investigated and the link between the rating formula and the insurer's solvency margin is not considered. 
Criterion (1) allows the weighting of variations in premiums and insurer's solvency margin. Moreover, the procedure to be introduced below gives for a fixed structure of the claims process also the corresponding optimal form of the rating formula. Thus criterion (1), together with the optimal rating rule, may also be of practical interest.

We further assume that the premiums have to be determined with $f$ years' time-lag in information; i.e., when the premiums $P(t)$ of the year $t$ are to be determined, the claims or equivalently the solvency margins at the end of the years $t-1-f, t-2-f, \ldots$ with $f \geqslant 0$ are known. Perhaps the most common value is $f=1$ i.e., the premiums for the year $t$ have to be calculated during the year $t-1$, when $X(t-1)$ is still unknown. But larger values are also interesting; e.g., when the IBNR reserves constitute a substantial part of $X(t)$.

The considerations are limited to the feedback rules. These rules are based on the observed solvency margins (or equivalently on the observed total claims amounts). The performance of the feedback rules might possibly be improved by adding a feedforward control, where the future outcomes of the claims process $X(t)$ are forecast (see e.g., Box-Jenkins, 1976, Chapter 12). Note, however, that in order to improve the feedback rule forecasts for the future $X(t)$ should be based on external factors: if the past of $X(t)$ only is used for this purpose the resulting procedure is again a feedback rule.

When transformed to a state-space model this problem can easily be attacked by using the famous Kalman filter technique. This algorithm was first presented by Kalman in 1960. Since then state-space models have been used in various areas ranging from engineering to econometrics, one of the reasons being that the Kalman filter equations are very easy to deal with on computers.

The idea of using (stochastic) control theory and Kalman filter in premium setting and actuarial calculations is not new. Pioneering works include e.g., Balzer-Benjamin (1980), (1982), Bohman (1979), DE Jong-Zehnwirth (1983), MARTIN-Lof (1983) and Ryder (1977).

In many important cases premiums cannot be totally controlled by the insurer but are determined e.g., by market conditions. Since in this paper we assume that the controller is in a position to set the premiums at the computed level, the results might most easily be applied in those insurance classes where the rates are subject to public control, or e.g., in long-term reinsurance agreements or in individually rated policies. But also for other cases the results may be of some interest, e.g., when the appropriate range for solvency margins are set up, or joint tariffs, based on the outcomes of the whole market, are used. Another interesting application would be for transfers into or from the contingency reserve to be calculated using formula (2.1) below, with risk premiums $P(t)$ given by an optimal control-theoretical rule (see also CHRISTENSEN, 1984, p. 181).

The basic model is introduced in Sections 2 and 3. The state-space form of the model is derived in Section 4. In Section 5 the optimal solution for the specific loss function (1) is given and Section 6 includes some numerical examples and an application to empirical data. 
2. THE SOLVENCY MARGIN PROCESS

The solvency margin (or accumulated profit) is assumed to progress according to equation

$$
U(t+1)=r(t) \cdot U(t)+P(t)-X(t)
$$

where in the year $t$

$$
\left\{\begin{aligned}
U(t) & =\text { solvency margin at the beginning of the year, } \\
r(t) & =\text { interest coefficient } \\
P(t) & =\text { risk premiums retained, } \\
X(t) & =\text { claims incurred on the insurer's own account. }
\end{aligned}\right.
$$

All these variables are in principle stochastic, but in order to keep the calculations simple $r(t)$ is taken as a non-random and time-independent constant $r$.

Note that equation (2.1) is the same for all values of delay parameter $f: f$ only regulates the information available for determination of $P(t)$.

Equation (2.1) is taken to include only the business retained. Thus in potential practical applications the rates resulting from the formulae below should be modified by a loading for reinsurance costs. Another interpretation is to take (2.1) to mean the gross business but let the division of premiums etc. between direct writer and reinsurer remain unspecified.

Since $U(t), \Delta^{d} X(t)$ and $\Delta^{d} P(t)$ denote the deviations from the corresponding expectations, then, in order to have a positive expectation for the solvency margin, another positive safety loading should be added to the premiums resulting from the rating rules below. A rational magnitude for the safety loading might be such that the expectation of $U(t)$ becomes a suitable multiple of the standard deviation of the $U(t)$.

\section{THE STRUCTURE OF THE CLAIMS PROCESS}

Introduce next the structure of the claims process. In time series theory much attention has been paid to so-called ARIMA models, especially since the publishing of the monograph by Box-Jenkins in 1970. However, the theoretical justification of such models was already given by WOLD (1938) and YAGLOM (1955). Recently such models have also received attention in articles on experience rating theory; cf. Jewell (1976), Sundt (1981), (1983) and Kremer (1982), (1983).

In an ARIMA model of $\operatorname{order}(p, d, q)$ a stochastic process $X(t)$ is described by the equation

$$
\Phi(B) \Delta^{d} X(t)=\Theta(B) a(t)
$$

where

$$
\left\{\begin{aligned}
\Phi(B)= & 1-\phi_{1} B-\phi_{2} B^{2}-\cdots-\phi_{p} B^{P} \\
\Theta(B)= & 1-\theta_{1} B-\theta_{2} B^{2}-\cdots-\theta_{q} B^{q} \\
B= & \text { backward shift operator; i.e., } B X(t)=X(t-1), \\
a(t)= & \text { a sequence of uncorrelated random variables } \\
& \text { with mean zero and with variance } \sigma_{a}^{2} .
\end{aligned}\right.
$$


If $d>0$, then the $X(t)$ process defined by (3.1) is non-stationary, but if the roots of equation $\Phi(B)=0$ lie outside the unit circle the $d$ th difference $\Delta^{d} X(t)$ of $X(t)$ is stationary.

In classical risk theory it is usually assumed that $p=d=q=0$ i.e., $X(t)$ is a white noise process. This assumption is hardly justified in practice unless the quantities are discounted for inflation. But also the discounted claims process may be expected at least in some insurance classes to be affected by jumps, trends, cycles etc., which means that the claims process is not white noise and can even be non-stationary. It is often possible adequately to describe these influences by ARIMA models. Unfortunately the relevant time series are usually far too short for any reliable estimation of parameters. An alternative is then to fix in advance the structure of $X(t)$ such that it can believed to be a cautious approximation to the processes occurring in practice. This is the logic behind the numerical examples in Section 6.

\section{THE SOLVENCY MARGIN PROCESS IN THE STATE-SPACE FORM}

In order to have access to the Kalman filter technique we have to transform equations (2.1) and (3.1) to a state-space model. A state-space model consists of two equations: the state equation describing the transition of the system and the observation equation giving the observable output of the system. In our case these equations are relatively simple to derive. We use the technique presented e.g., in HARVEY (1981).

Take first the state equation. From (2.1) and (3.1) we obtain

$$
(1-r B) \Phi(B) \Delta^{d} U(t+1)=\Phi(B) \Delta^{d} P(t)-\Theta(B) a(t) .
$$

Let $n=\max (p+d+1, q+1)$. Assume first that the delay parameter $f=0$. Introduce $n$ state variables $Z(1, t), Z(2, t), \ldots, Z(n, t)$. A state equation corresponding to $(4.1)$ is

$$
Z(t+1)=A Z(t)+G \Delta^{d} P(t)-M a(t)
$$

where

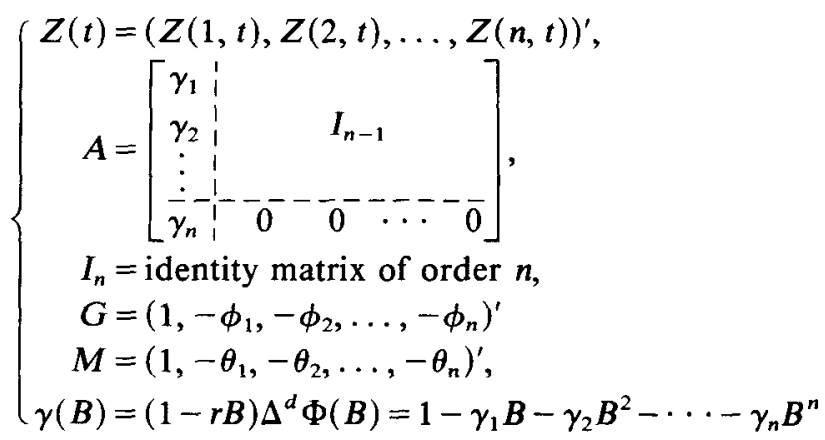

with $\phi_{i}=0$ for $i>p$ and $\theta_{i}=0$ for $i>q$ and 'denotes transposition.

The solvency margin $U(t)$ is now given as the first element of $Z(t)$. Since, according to assumption that $f=0, U(t)$ (and thus $Z(t)$ ) is known when the 
premiums for year $t$ are to be determined, an observation equation is now not needed; i.e., we have the case of complete state information (see ÅströM, 1970, Section 8.4).

The equivalency of (2.1) and (3.1) to (4.2) can be verified by a straightforward calculation. Note, however, that the state-space representation is not unique.

A positive time delay $f$ may be taken into account by the addition of $f$ new state variables of the form

$$
\left\{\begin{aligned}
Z(n+1, t) & =Z(1, t-1) \\
Z(n+2, t) & =Z(n+1, t-1) \\
\vdots & \\
Z(n+f, t) & =Z(n+f-1, t-1)
\end{aligned}\right.
$$

and by modifying $A, G$ and $M$ respectively. Moreover, we now also need the observation equation of the form:

$$
Y(t)=H Z(t),
$$

where $H=\left(\begin{array}{llll}0 & 0 & \ldots 1\end{array}\right)$ is a $(1 \times n+f)$ vector. Thus $Y(t)=U(t-f)$.

Note that the solvency margin $U(t)$ is still given as the first element of $Z(t)$. However, its value can only be calculated after $f$ years time; i.e., the total claims amounts for the years $t-1, t-2, \ldots, t-1-f$ are not yet known in the year $t-1$.

\section{THE OPTIMAL RATING EQUATIONS}

Consider a finite planning horizon consisting of years $s, s+1, \ldots, N$. The loss function (1) is a special case of the loss function

$$
E\left\{Z(N)^{\prime} Q_{0} Z(N)+\sum_{t=s}^{N-1}\left[Z(t)^{\prime} Q_{1} Z(t)+v \cdot\left(\Delta^{d} P(t)\right)^{2}\right\}\right.
$$

where $Q_{0}$ and $Q_{1}$ are symmetric positive definite $(n+f) \times(n+f)$-matrices.

The optimal linear control rule giving the minimum for this loss function is (see e.g., Åström, 1970, Theorem 4.1 in Section 7.4 and Theorem 5.1 in Section 8.5):

$$
\Delta^{d} P(t)=-L(t) \hat{Z}(t)
$$

where $\Delta^{d} P(t)$ is the optimal premium setting to be applied at time $t$, and $\hat{Z}(t)$ is the least squares estimator of the state vector $Z(t)$ when $Y(t), Y(t-1), \ldots$ and the prior distribution of $Z(s)$ is the data available for determining the control action. $L(t)$ is a $(1 \times n+f)$ vector of constants given by

$$
L(t)=\left[v+G^{\prime} S(t+1) G\right]^{-1} G^{\prime} S(t+1) A,
$$

where $S(t+1)$ is obtained from recurrence equation

$$
S(t)=A^{\prime} S(t+1) A+Q_{1}-A^{\prime} S(t+1) G L(t)
$$

with the initial condition

$$
S(N)=Q_{0}
$$


The state estimator $\hat{Z}(t)$ for the undelayed system equals $Z(t)$. For the delayed case $\hat{Z}(t)$ can be obtained from the Kalman filter equations (see $\AA$ sтröM, 1970, Exercise 7 on p. 235):

$$
\begin{aligned}
& \hat{Z}(t)=\hat{Z}_{1}(t)+K(t)\left[Y(t)-H \hat{Z}_{1}(t)\right] \\
& K(t)=R_{1}(t) H^{\prime}\left[H R_{1}(t) H^{\prime}\right]^{-1} \\
& R(t)=R_{1}(t)-K(t) H R_{1}(t) \\
& \hat{Z}_{1}(t)=A \hat{Z}(t-1)+G \Delta^{d} P(t-1)
\end{aligned}
$$

and

$$
R_{1}(t)=A R(t-1) A^{\prime}+\Sigma_{1}
$$

where $\Sigma_{1}=\sigma_{a}^{2} \cdot M M^{\prime}$ the recursion starts with $\hat{Z}(s)=\mu_{0}$ and $R(s)=\Sigma_{0}$ where $\mu_{0}$ and $\Sigma_{0}$ are the prior expectation and covariance matrix of $Z(s)$, respectively, and with $\Delta^{d} P(s)=-L(s) \mu_{0}$. The starting values for $P(t)$ and $Y(t)=$ $U(t-f)(t<s)$ are the actual ones.

Thus the optimal procedure depends on the chosen prior values $\mu_{0}$ and $\Sigma_{0}$. However, one is often interested in an asymptotic or steady-state solution (i.e., a solution where the transient effects due to chosen prior values have died out). It can be shown that as $N \rightarrow \infty$ the matrices $R(t)$ and $S(t)$ will converge to unique steady-state positive definite values $R$ and $S$. Denote the corresponding limits of $L(t)$ and $K(t)$ by $L$ and $K$. Then the steady-state feedback rating formulae are

$$
\begin{gathered}
\Delta^{d} P(t)=-L \hat{Z}(t), \\
\hat{Z}(t)=\left(I_{n+f}-K H\right)(A-G L) \hat{Z}(t-1)+K Y(t),
\end{gathered}
$$

for $f \geqslant 1$. In the undelayed $f=0$ case the latter equation is again replaced by $\hat{Z}(t)=Z(t)$. These equations are quite easy to translate into a more traditional form involving only past $\Delta^{d} P(t): s$ and $U(t): s$ or $X(t): s$. Some examples are given in Section 6.

The corresponding steady-state covariance matrix $C_{Z}$ of the state vector $Z(t)$ can be obtained by iteration from equation:

$$
C_{Z}=(A-G L)\left[C_{Z}(A-G L)^{\prime}+R L^{\prime} G^{\prime}\right]+G L R A^{\prime}+\Sigma_{1} .
$$

The steady-state variance of the solvency margin is given as the left upper corner element of $C_{Z}$.

The corresponding variance of $\Delta^{d} P(t)$ is

$$
\operatorname{Var}\left(\Delta^{d} P(t)\right)=L\left(C_{Z}-R\right) L^{\prime} .
$$

Note that when $d>0$ the variance of the premiums is infinite $(X(t)$ also has infinite variance) but the variance of the solvency margin is finite.

Numerical calculation by computer of the steady-state solution is quite easy since equations (5.3), (5.4), (5.7), (5.8) and (5.10) do not depend on observations and the solution can be obtained by successive iteration. 
It is also possible to derive the above steady-state optimal rating procedures in a more easily interpretable way using the technique presented in Box-JENKINS (1976), Section 13.2 (see Rantala 1984). This technique, however, may lead to computational difficulties when the orders of operators $\Phi(B)$ or $\Theta(B)$ in (3.1) are not low. On the other hand, the Kalman filter technique is in essence independent of the order of $X(t)$-process, only the dimension of the transformation (4.2)-(4.5) depends on the order parameters $p, d$ and $q$. But the method presented in Box-Jenkins is also applicable when, for example, the steady-state variance of $\Delta P(t)$ is to be minimized when $X(t)$ is a stationary process, whereas the loss function (5.1) applies only to the variance of $P(t)$.

\section{EXAMPLES}

Next some numerical examples are given of the application of the asymptotic procedures introduced in Section 5.

Example 1. Assume first that there is some variable $V(t)$ which may serve as a basic volume measure. For example, $V(t)$ may be the sums insured in fire insurance, the payroll in workers' compensation insurance etc. Usually $V(t)$ is also stochastic, but since the main variation of $X(t) / V(t)$ is obviously due to $X(t), V(t)$ is treated here as a deterministic quantity. Our assumptions are

(a) the quantities of $(2.1)$ in the year $t$ are proportional to the basic volume measure $V(t)$ (and we call them rates),

(b) $\Delta X(t)=a(t)-\theta a(t-1)$; i.e., $X(t)$ is an ARIMA $(0,1,1)$ process.

Assumption (a) means that now $r=r_{i} / r_{g}=$ interest coefficient $r_{i}$ divided by the growth factor $r_{g}$ of $V(t)$. Note that $r_{g}$ includes both real and inflation growth of the volume. Assumption (b) allows the interpretation that every year a shock $a(t)$ is added to the current "basic level" of the claims rate to produce a value $X(t)$. However, only a proportion $1-\theta$ of the shock $a(t)$ is actually absorbed into the new basic level to have lasting influence (see BoX-JENKINs, 1976, Chapter 4).

In practice perhaps not every shock changes the level. Thus (b) may be regarded as a cautious "upper limit" for actual claims processes. When $\theta=0$ we obtain a random walk process; i.e., every new shock is totally absorbed into the basic level, this being the "most dangerous" alternative among the $\operatorname{ARIMA}(0,1,1)$ processes.

We want a rating rule which minimizes the steady-state variance of $\Delta P(t)$ subject to a constraint on the steady-state variance of $U(t)$. This can be done by choosing the loss function as

$$
Q_{0}=Q_{1}=\left[\begin{array}{ll}
w & 0 \\
0 & 0
\end{array}\right]
$$

and $v=1$. By varying $w$ different combinations of $\operatorname{Var}(\Delta P(t))$ and $\operatorname{Var}(U(t))$ can be produced from which a suitable compromise may be found. As an example 
we take $r=1, \theta=0$ and $w=0.005$. By applying the iterative equations of Section 5 we obtain the optimal steady-state rating rule simply as

$$
\Delta P(t)=-0.373 \cdot U(t)+0.314 \cdot U(t-1) .
$$

Formula (6.2) can also be expressed in terms of previous $X(t): s$ using equation (2.1). Straightforward calculation shows that (6.2) is equivalent to

(6.3) $P(t)=1.627 \cdot P(t-1)-0.686 \cdot P(t-2)+0.373 \cdot X(t-1)-0.314 \cdot X(t-2)$.

The corresponding variances turn out to be

$$
\left\{\begin{array}{l}
\operatorname{Var}(U(t))=27.66 \sigma_{a}^{2} \\
\operatorname{Var}(\Delta P(t))=0.32 \sigma_{a}^{2}
\end{array}\right.
$$

In fact, rule (6.2) corresponds to the "integral controller" introduced in BALzERBenjamin (1980), see also BoX-Jenkins (1976), Section 12.2.2. It shows that in this case the use of $U(t)$ only (which is equivalent to the traditional exponential smoothing formula for experience rating) is not enough to produce an optimal scheme.

Since the underlying claims process used in the derivation of (6.2) is assumed to be a random walk, procedure (6.2) is able to detect every persistent jump in the claims process; i.e., even if $X(t)$-process has a persistent jump to a new level $X_{0}$ the steady-state values of both $E\left(P(t)-X_{0}\right)$ and $E U(t)$ are zero. The corresponding steady-state value of $E U(t)$ for traditional exponential smoothing is non-zero (see also BALZER-BenJAMin (1980) and (1982)).

Example 2 (continued). We take the model of Example 1 with the same numerical values for other parameters but assume that $f=1$; i.e., the solvency rate $U(t)$ is not known when the premium rates for the year $t$ are to be determined. Now the optimal steady-state rule is

$$
\Delta P(t)=-0.373 \cdot \Delta P(t-1)-0.431 \cdot U(t-1)+0.373 \cdot U(t-2) .
$$

Thus the change in the premiums in the year $t$ now depends, besides on two latest observed solvency rates, also on the previous change itself.

The variance calculations give

$$
\left\{\begin{array}{l}
\operatorname{Var}(U(t))=51.4 \sigma_{a}^{2} \\
\operatorname{Var}(\Delta P(t))=0.4 \sigma_{a}^{2} .
\end{array}\right.
$$

The following figure shows the possible optimum combinations of steady-state $\operatorname{Var}(U(t))$ and $\operatorname{Var}(\Delta P(t))$ in Examples 1 and 2. Moreover, Example 4 gives a practical application of a rule like (6.5).

As expected, the variances for the case $f=1$ are somewhat larger than for the case $f=0$. However, the increases are only moderate. It can also be seen that reduction of the standard deviation of $\Delta P$ below $0.5 \sigma_{a}$ entails a rapid increase in the standard deviation of $U$.

Note also the superiority of the above optimal scheme over the "naive" strategy $P(t)=X(t-1)$ (when $f=0$ ); i.e., the claims of the preceding year are paid by 


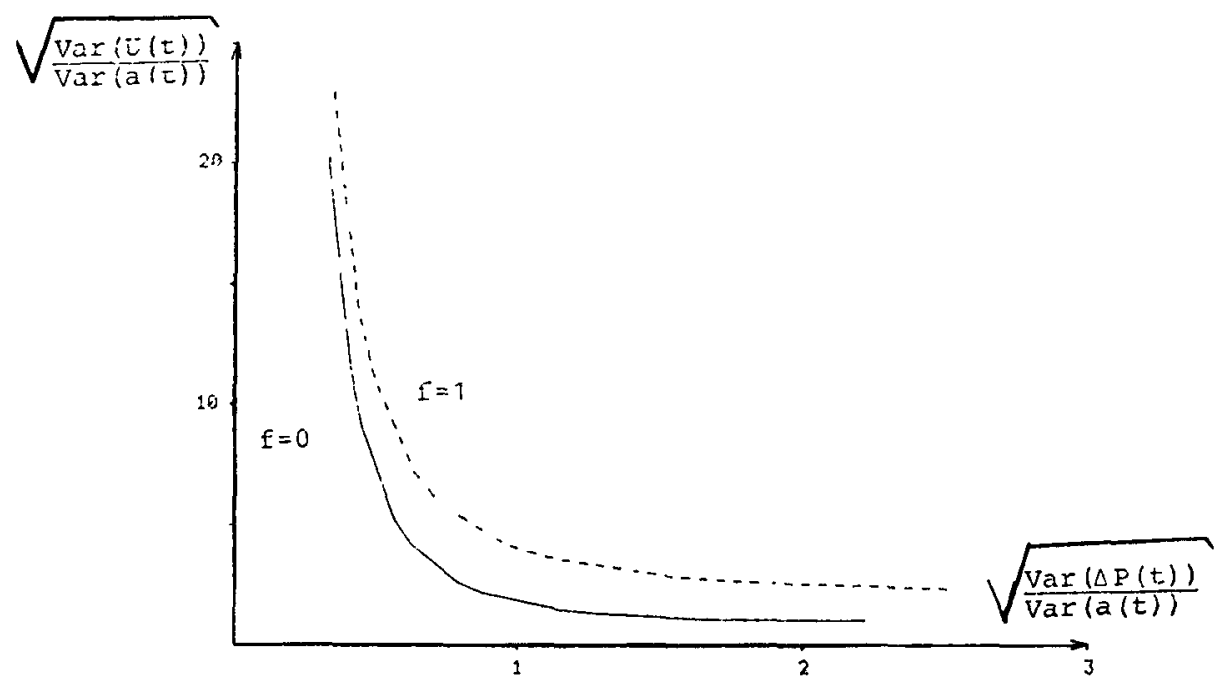

FIgURE 6.1. The optimal combinations of $\operatorname{Var}(U(t))$ and $\operatorname{Var}(\Delta P(t))$ in Examples 1 and 2.

the policy-holders in the next year. This is also the "pure" credibility rating rule for the random walk claims process; i.e., the premiums are set equal to the "best" predictor of the total claims amount of the following year without regard to the amount of the accumulated profit. By this policy the steady-state standard deviation of the solvency rate would be infinite and the steady-state standard deviation of the change in the premium rate would be a $\sigma_{a}$. However, using the above optimal scheme this latter variance can be achieved with the standard deviation of the solvency rate at only $1.6 \sigma_{a}$.

As noted, procedures (6.2) and (6.5) are able to detect permanent jumps in the claims process, while linear trends pass unnoticed. The procedure of example 3 is designed to deal with such trends.

Example 3. Now it is assumed that no basic volume measure is available and the quantities in (2.1) are absolute amounts, which among other things are subject to the influence of inflation. Then a cautious approximation analogous to Example 1 is:

$$
\Delta^{2} X(t)=a(t)-\theta_{1} a(t-1)-\theta_{2} a(t-2)
$$

i.e., $X(t)$ is an ARIMA $(0,2,2)$ process. It can be shown (see e.g., BoX-JENKINS, 1976 , Chapter 5) that for a series generated by (6.7), the optimal forecasts (in the mean square error sense) lie along a straight line, the level and slope of which are continually updated as new data become available. In fact, in equation (6.7) $1+\theta_{1}+\theta_{2}$ and $1-\theta_{2}$ are the fractions of the shock $a(t)$ which are transmitted to the level and slope parameters, respectively. Thus, model (6.7) may be interpreted to include the effect of inflation with permanent changes in the inflation rate 
every year, this being a cautious approximation to the inflations occurring in practice.

As a numerical example take $r=1.1$ and $\theta_{1}=-0.5, \theta_{2}=1$. It is further assumed that $f=1$.

Let again $v=1$ and

$$
Q_{0}=Q_{1}=\left[\begin{array}{cccc}
0.005 & 0 & 0 & 0 \\
0 & 0 & 0 & 0 \\
0 & 0 & 0 & 0 \\
0 & 0 & 0 & 0
\end{array}\right]
$$

A straightforward calculation gives the optimal rule as

$$
\left(1+0.418 B+0.384 B^{2}\right) \Delta^{2} P(t)=\left(-1.424+2.417 B-1.059 B^{2}\right) U(t-1)
$$

As compared to example 2 we have here the second difference of $P(t)$ and one more time lag on both sides of the rating equation.

The corresponding steady-state variances are

$$
\left\{\begin{array}{l}
\operatorname{Var}(U(t))=218 \sigma_{a}^{2}, \\
\operatorname{Var}\left(\Delta^{2} P(t)\right)=2.95 \sigma_{a}^{2} .
\end{array}\right.
$$

In interpreting these variances it should be observed that here $\sigma_{a}^{2}$ is independent of the level of the $X(t)$ process. In practice inflation obviously also increases the variance in the nominal claims amount. In any case, this example shows that the optimal smoothing of the claims process with stochastic linear trends obviously requires a rather complicated rating rule.

Example 4. Third party motor liability insurance in Finland 1966-1982 is taken as an empirical example. The annual claims and premiums are computed per one "reduced policy year", the durations and bonus reductions of policies being taken into account in that e.g., a bonus of $60 \%$ and duration of one year correspond to a reduced policy year of length 0.4 . The premiums are computed on an earned basis. Both claims and premiums are discounted to FIM (1982) using the average of living costs and salary index. Thus claims, premiums and solvency margin may be taken to correspond to the "rates" introduced in Example 1. The combined increase in index and reduced policy years has been on the average $13.8 \%$, and since the interest yield of the acccumulated profit is not credited to surplus, the average interest coefficient $r=0.8785$ (see Example 1). The risk premiums are obtained by deducting expenses from gross premiums. The claims for each year are those estimated at the end of 1982 . Thus they are not quite the same as those used in actual premium setting.

We compare the rates emerging from the rating rule

$$
\Delta P(t)=-0.2765 \cdot \Delta P(t-1)-0.3042 \cdot U(t-1)+0.2429 \cdot U(t-2)
$$

to the actual rates. Rule (6.10) is in the following called random walk rating, since it gives an optimal combination $\sqrt{\operatorname{Var}(U(t))}=6.3 \sigma_{a}$ and $\sqrt{\operatorname{Var}(\Delta P(t))}=$ $0.477 \sigma_{a}$ if $X(t)$ is a random walk and with one year's time delay in information. 


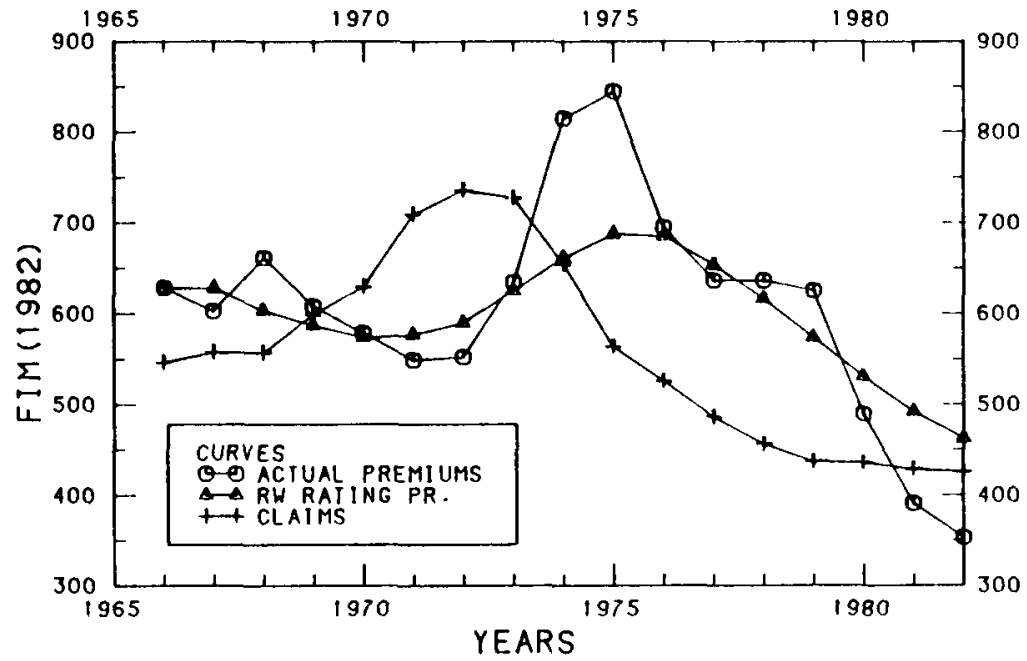

FIGURE 6.2. The claims, actual premiums and random walk premiums per one "reduced policy year" in FIM (1982) in Finnish third party motor liability 1966-1982.

In applying (6.10) the initial solvency rate is set equal to zero and the initial premium equal to the actual value.

Figures 6.2 and 6.3 show the rates and the accumulated surpluses of the procedures. The annual changes in premiums are given in fig. 6.4.

It may be concluded that the random walk rating would have led to somewhat smaller fluctuations in accumulated profits and to considerably smoother changes in premiums than the actual rating. However, it should be remembered that there

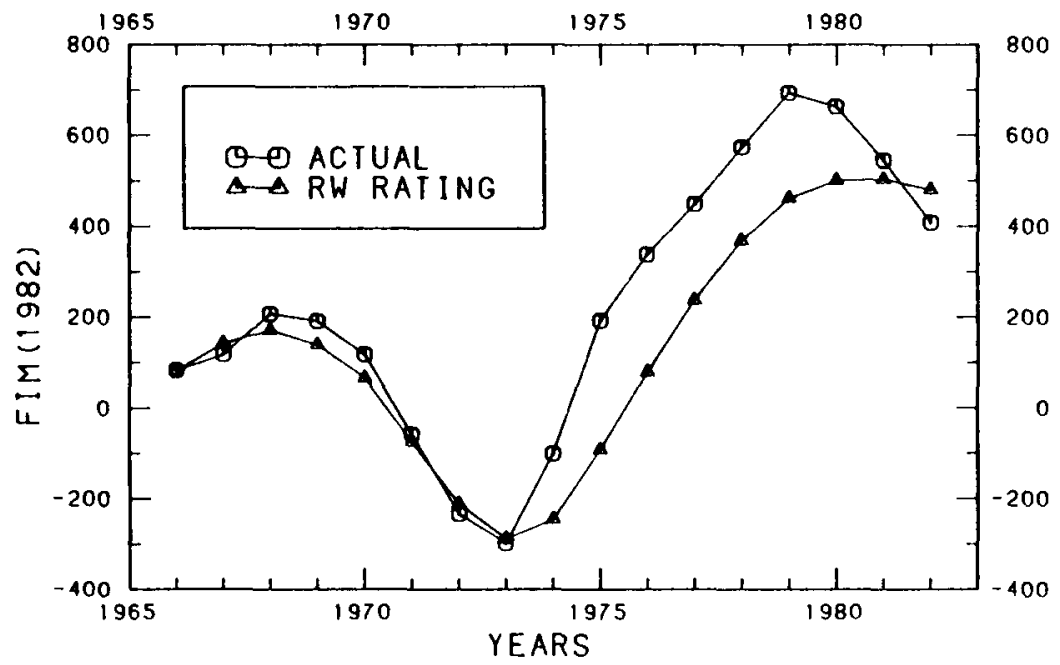

FIGURE 6.3. The accumulated profits from fig. 6.2. 

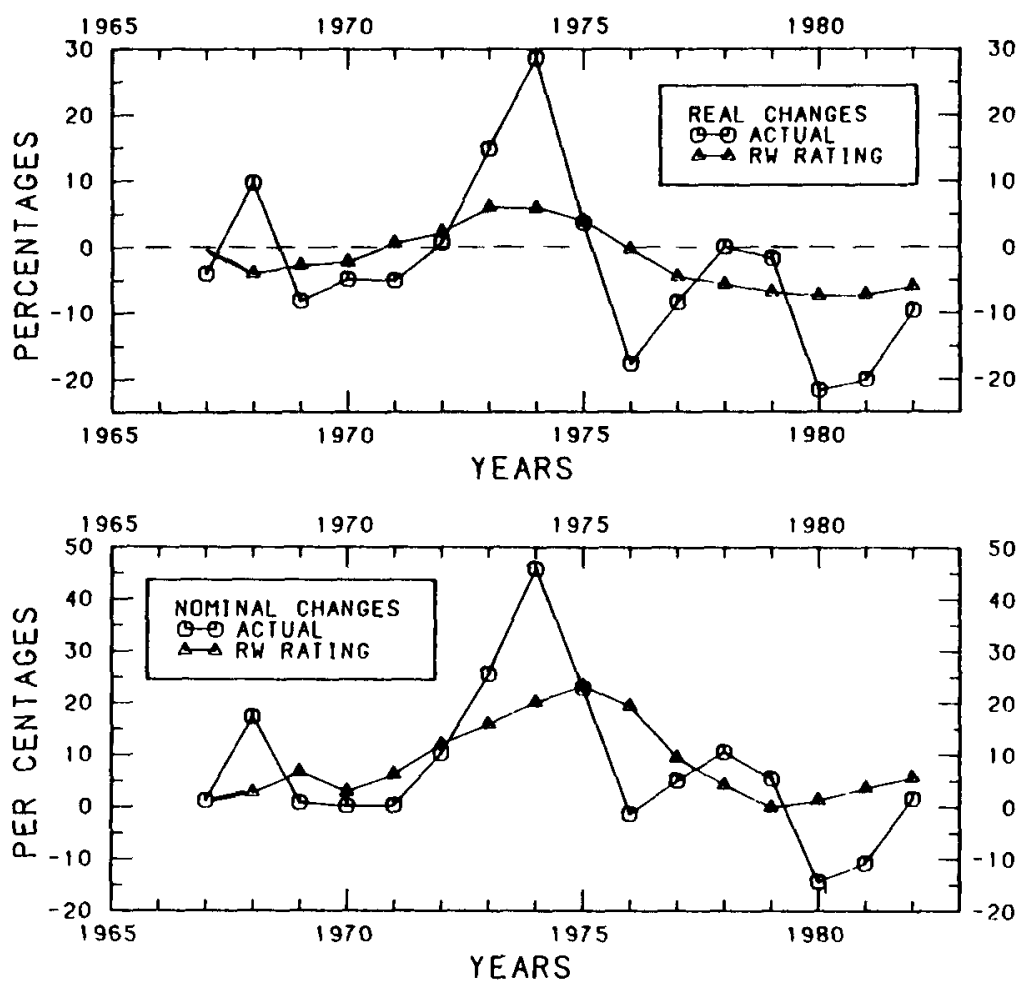

FIGURE 6.4. The annual relative changes from fig. 6.2 in premiums in real terms (upper figure) and in nominal values (lower figure).

may be other background factors than only actuarial for the performance of the actual scheme; e.g., the general price freeze in the late 60 's after which there was a sudden increase of premiums in 1973-1974. That increase and the simultaneous decrease in claims led to a rapid increase in accumulated profit. In any case, it seems that the theoretical random walk rating (with suitable parameters) does not lead to too rapid reactions, and at least in this example its performance is fairly satisfactory. Moreover, as noted in the introduction, its performance could still be improved if an "outside" forecast of the claims were added (see e.g., the sudden increase in claims at the beginning of the 70's and a subsequent steep decrease).

\section{CONCLUDING REMARKS}

This paper is intended to illustrate how the methods of stochastic control theory can be used in experience rating. The explicit model considered is a simplified picture of real phenomena and there remain many interesting problems to solve when the model is enlarged to greater detail. For example, the effects of run-off errors on the optimal control rules can be studied in the Kalman filter framework by adding a noise term on the righthand side of the observation equation (4.5) to approximate these errors. 
In addition to the variance criterion, the performance of these rules may be studied e.g., by examining their time responses to jumps, steps and linear trends in the claims process. By studying the frequency response functions of the premium and solvency margin filters resulting from these rules it is also possible to obtain an image of their behaviour in cases where the claims process is not that assumed in the derivation of the rules. In this way it is also possible to obtain upper limits for the variances, independent of the actual correlation structure of the claims process. Some of these questions are treated in RANTALA (1984).

\section{ACKNOWLEDGEMENT}

The author wishes to express his thanks to the referees for many valuable comments which led to improvements in the presentation of this paper.

\section{REFERENCES}

BAlzer, L. A. and Benjamin, S. (1980) Dynamic Response of Insurance Systems with Delayed Profit/Loss Sharing Feedback to Isolated Unpredicted Claims. Journal of Institute of Actuaries 107, 513-529.

Balzer, L. A. and Benjamin, S. (1982) Control of Insurance Systems with Delayed Profit/Loss Sharing Feedback and Persisting Unpredicted Claims. Journal of Institute of Actuaries 109, 285-316.

BонмаN, H. (1979) Insurance Economics. Scandinavian Actuarial Journal 57-74.

Box, G. E. P. and Jenkins, G. M. (1976) Time Series Analysis: Forecasting and Control. Holden Day.

Christensen, I. E. (1984) On the Use of Contingency Reserves in Surplus Allocation. Transactions of the 22nd International Congress of Actuaries.

DE JoNG, P. and ZEHNwIRTh, B. (1983) Claims Reserving, State-Space Models and the Kalman Filter. Journal of Institute of Actuaries 110, 157-182.

Harvey, A. C. (1981) Time Series Models. Allan Phillips Publisher Limited: Oxford.

Jewell, W. S. (1976). Two Classes of Covariance Matrices Giving Simple Linear Forecasts. Scandinavian Actuarial Journal 15-29.

Kalman, R. E. (1960) A new approach to linear filtering and prediction problems. Trans. Amer. Soc. Mech. Eng., I. Basic Engineering 82.

KREMER, E. (1982) Credibility Theory for Some Evolutionary Models. Scandinavian Acturial Journal, 129-142.

Kremer, E. (1983) A Remark on Parameter-Estimation of Autoregressive Credibility-Models. Deutsche Gesellschaft für Versicherungsmathematik XVI, 153-160.

MARTIN-Löf, A. (1983) Premium control in an Insurance System, an Approach Using Linear Control Theory. Scandinavian Actuarial Journal 1-28.

Rantala, J. (1984) An Application of Stochastic Control Theory to Insurance Business. Ph.D. Thesis, Department of Mathematical Sciences, University of Tampere.

Ryder, J. M. (1977) Predicting Future Premiums-Theory and Practice. General Insurance Bulletin $460-483$.

SUNDT, B. (1981) Recursive Credibility Estimation. Scandinavian Actuarial Journal 3-21.

SUNDT, B. (1983) On Time-Heterogeneous Credibility Estimation. Scandinavian Actuarial Journal 183-190.

ÅströM, K. J. (1970) Introduction to Stochastic Control Theory. Academic Press.

Wold, H. (1938) A Study in the Analysis of Stationary Time-Series. Almqvist \& Wicksell: Stockholm, 2nd ed. 1954.

Yaglom, A. M. (1955) The Correlation Theory of Processes whose $n$th Difference Constitutes a Stationary Process. Matem. Sb. 141.

JukKa Rantala

Ministry of Social Affairs and Health, Insurance Department, Bulevardi 28, SF00120 , Helsinki 12. 